\title{
Covalently capped seed-mediated growth: a unique approach toward hierarchical growth of gold nanocrystals $\uparrow$
}

Received 30th January 2014 Accepted 5th April 2014

DOI: $10.1039 / c 4 n r 00587 b$

www.rsc.org/nanoscale

Hierarchical metal nanostructures have attracted increasing interest due to their unique morphology-dependent properties. Here, we introduce a new and efficient method to synthesize hierarchical gold nanostructures in different shapes using the covalently capped seedmediated growth approach.

Over the last decade, great efforts have been devoted to the development of nanostructures with different morphologies which considerably influence their intrinsic properties and relevant applications. In this regard, noble metal nanoparticles (NPs), especially gold ( $\mathrm{Au}$ ), have drawn most attention because of its unique properties (physical, chemical and biocompatible) and promising applications, including selective catalysis, ${ }^{1-3}$ biosensing, ${ }^{4-7}$ nanomedicine, ${ }^{8,9}$ information storage, ${ }^{10}$ and optical imaging. ${ }^{11-15}$ Among various shapes and morphologies, our interest is focused on the development of 3D hierarchical nanostructures because of their rough surface and large surface-to-volume ratio ${ }^{16-18}$ which contribute to superior surface-sensitive properties in catalysis and surface-enhanced Raman scattering (SERS) compared to low dimensional structures..$^{17-20}$

Despite recent progress in synthetic methods, it still remains a great challenge to synthesize hierarchical Au nanostructures of uniform size with a high yield and good reproducibility. In general, the seed-mediated growth (also called seeded growth) method for the synthesis of NPs is regarded as the most efficient method for the precise growth control over the size and shape of NPs. However, NPs synthesized via the seeded growth approach $^{21}$ often result in polyhedral shaped (e.g. cube, octahedron, truncated octahedron, and icosahedron) or relatively less branched $\mathrm{Au}$ nanostructures ${ }^{22-24}$ as compared to that

School of Chemical and Biomedical Engineering, Nanyang Technological University, 637457, Singapore. E-mail: dhkim@ntu.edu.sg; Fax: +65 6791 1761; Tel: +65 67904111

$\dagger$ Electronic supplementary information (ESI) available: Experimental details and Fig. S1-S8 as mentioned in the text. See DOI: 10.1039/c4nr00587b

$\ddagger$ These authors have contributed equally to this work. obtained via a single step method ${ }^{25}$ or a galvanic replacement method. ${ }^{26}$ The key challenge for hierarchical growth of fcc metals (e.g. Au and $\mathrm{Ag})$ in the seeded growth approach comes from the fact that the process occurs at a slow growth rate (i.e. the rate of adatom adsorption on a seed surface). ${ }^{27}$ During the course of growth, the adatom adsorption process is accompanied by a migration process that allows the diffusion of adatoms to the low energy facets of the crystal. When the rate of adatom diffusion is faster than that of adsorption, thermodynamically stable polyhedra would be favoured (Scheme 1a; case 1). Conversely, when the adsorption of adatoms occurs at a faster rate than its diffusion, growth becomes kinetically mediated, leading to the formation of highly branched nanostructures (Scheme 1a; case 2). ${ }^{28,29}$ However, if the growth rate exceeds a critical value ( $R_{\text {critical }}:$ an optimum growth rate where selfnucleation is suppressed), the uncontrolled formation of small particles will occur, making it difficult to maintain the uniformity in size and shape (Scheme 1a; case 2). ${ }^{27,30}$ Thus, we
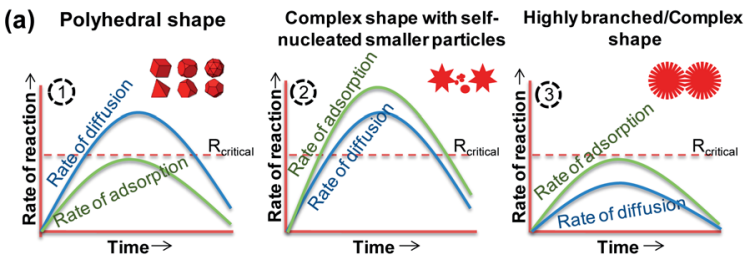

(b)

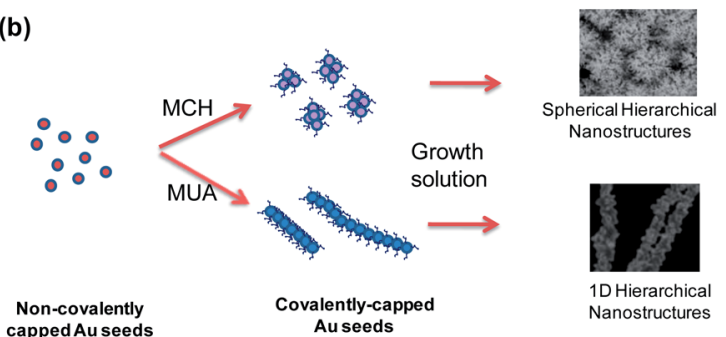

Scheme 1 Schematic illustration of the formation of a hierarchical Au nanostructure. 
hypothesize that this problem can be solved by increasing the bonding strength between the capping agents and the metal surface to impede the diffusion process (Scheme 1a; case 3). The slow diffusion of adatom would facilitate the formation of a highly branched hierarchical structure, retaining morphological uniformity. Most of the solution-based methods for hierarchical $\mathrm{Au}$ nanostructure synthesis reported previously describe the formation of a nearly spherical or flower-like structure. $^{23-25}$ Although $1 \mathrm{D}$ porous hierarchical Au nanostructures (e.g. wires, tubes or belts) were recently reported via template approaches or lithography techniques, ${ }^{31-33}$ there is still lack of a common and easy approach for the synthesis of hierarchical nanostructures across different shapes, i.e., from a sphere to a wire.

The shape acquired by a nanocrystal in a seeded growth approach is the consequence of numerous experimental parameters, ${ }^{22,34-37}$ of which the two most important factors are the initial shape of Au-seeds and the capping effects provided by the molecules or ions present in a growth solution. The use of different capping agents may alter the ratio of growth rates of different crystallographic planes within the NPs, resulting in the formation of different shapes of nanostructures. Most of the literature reports regarding the synthesis of $\mathrm{Au}$ nanostructures have been utilizing capping agents such as CTAB and citrate, which interact with the metal NP surface through van der Waals force. This non-covalent interaction allows a dynamic exchange between the free surfactants present in a growth solution and the surfactants attached to the seed surface during the growth process. This dynamic process contributes to relatively fast adatom diffusion, resulting in the formation of thermodynamically favoured structures. In this report, a non-covalently capped seed is replaced by a covalently capped seed to increase the bonding strength of the capping agents to the metal surface and slower down the adatom diffusion process. In this way, adatom adsorption is encouraged to occur at a faster rate than its diffusion, thus, ultimately favours hierarchical growth.

The seed-mediated approach that utilizes covalently modified seeds allows their overall shape to be tuned from a spherical- to a wire-like structure. So far, this is the first approach to synthesize both nearly spherical and 1D hierarchical Au nanostructures in a single, wet-chemical approach. Capping agents were chosen to play dual roles; firstly to form a covalent bond (through the -SH group) with the seed surface for hierarchical growth, and secondly to guide the overall shape of synthesized particles. 6-Mercaptohexanol $(\mathrm{MCH})$ having the propensity to form spherical agglomerates (Fig. S1, ESI $\dagger$ ) was chosen to grow a nearly spherical, flower-like 3D hierarchical nanostructure, whereas 11-mercaptoundecanoicacid (MUA) having the ability to assemble NPs into a chain-like structure ${ }^{38}$ (Fig. S1, ESI $\dagger$ ) was used to create a 1D hierarchical nanostructure as shown in Scheme 1(b).

An optimized solution containing a mixture of $2.5 \times 10^{-4} \mathrm{M}$ $\mathrm{HAuCl}_{4}, 0.01 \mathrm{M} \mathrm{CTAB}$ and $5.5 \times 10^{-4} \mathrm{M}$ ascorbic acid was used as a growth solution because it was found that this growth solution could effectively suppress self-nucleation (i.e., maintaining the adsorption rate lower than $\left.R_{\text {critical }}\right) \cdot{ }^{36,39-41}$ When a solution of $0.5 \mathrm{ml} \mathrm{MCH}$-modified $18 \mathrm{~nm}$ spherical Au seeds was allowed to grow in $10 \mathrm{ml}$ of the optimized growth solution, highly uniform 3D hierarchical nanostructures (HAuNSs) of size $\sim 400 \mathrm{~nm}$ was obtained as presented in Fig. 1a. The magnified image (Fig. 1b) of the particles clearly unveils highly branched and porous structures. High uniformity in the size of $\mathrm{Au}$ nanostructures gives evidence to the restriction of self-nucleation. By changing the $\mathrm{MCH}$ concentrations, we found that high concentrations of $\mathrm{MCH}$ tend to show similar hierarchical structures as shown in Fig. 1a, whereas low $\mathrm{MCH}$ concentrations produced slightly non-uniform hierarchical structures (Fig. S2, ESI†).

To understand the effect of the primary shape of the seeds on the final morphology, MCH-capped cubic seeds were allowed to grow in an identical growth solution. The resultant structure (Fig. S3, ESI $\dagger$ ) was similar to that obtained from the spherical seeds. This indicates that, unlike the conventional non-covalently capped seeded growth (Fig. S4, ESI $\dagger$ ), ${ }^{36}$ covalently capped seeded growth offers flexibility in the selection of primary seed shapes as the seed shape has little effect on the final morphology under identical growth conditions. Other surfactants containing the - $\mathrm{SH}$ group tested in the present study (i.e., 11-mercaptoundecanol, 1-nonanethiol, cysteamine and methoxy-poly(ethylene glycol)-thiol) also formed spherical hierarchical nanostructures under the same growth conditions (Fig. S5, ESI $\dagger$ ).

Next, MUA was covalently modified on the seed surface and allowed to grow under the identical growth conditions to that of MCH-modified seeds. Highly branched hierarchical nanowires (HAuNWs) were formed by spherical seeds of $40 \mathrm{~nm}$ (Fig. 1c and d), while moderately roughened hierarchical nanowires were obtained by a smaller spherical seed (18 nm) (Fig. S6, ESI $\dagger$ ). By changing the MUA concentrations, we found that high concentration of MUA produced nanowires with some agglomerated seeds remained as impurities (Fig. S7b, ESI $\dagger$ ) and
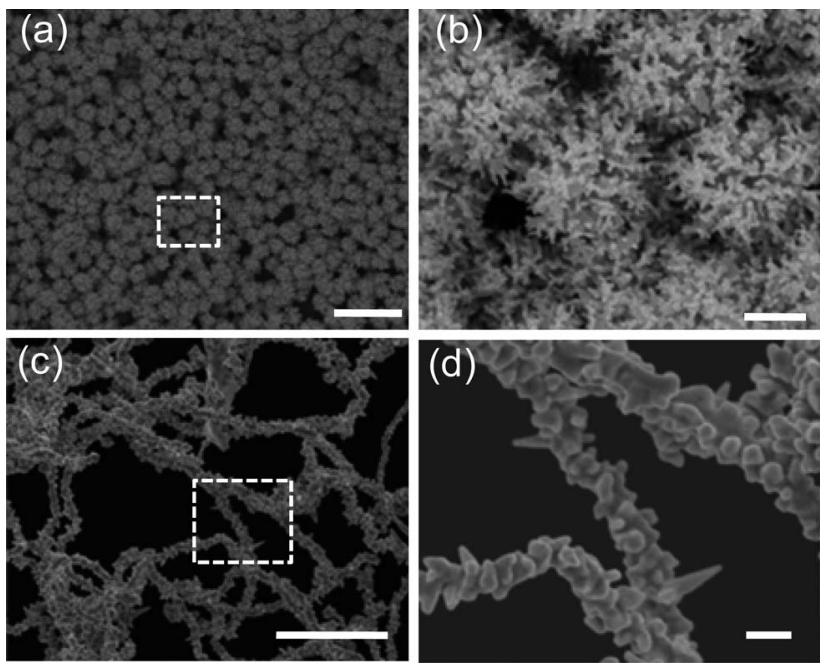

Fig. 1 SEM images of hierarchical Au nanostructures: ( $a$ and $b$ ) HAuNSs and ( $c$ and d) HAuNWs synthesized from growth of $\mathrm{MCH}$ - and MUA-modified Au seeds. The scale bars in the images $a, c$ and b, $d$ are $2 \mu \mathrm{m}$ and $200 \mathrm{~nm}$, respectively. 

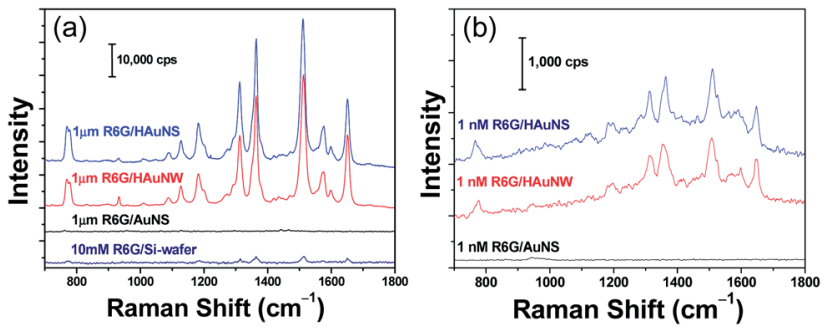

Fig. 2 SERS spectra of R6G adsorbed on Au nanostructures. The spectra were obtained with $\lambda_{\mathrm{ex}}=632.8 \mathrm{~nm}$ excitation.

low MUA concentration resulted in highly branched leaf like structures (Fig. S7a, ESI $\dagger$ ). To generalize the method to obtain hierarchical nanowires, 3-mercaptopropionic acid having similar end functional groups $(-\mathrm{COOH})$ was used to modify the Au seeds to achieve similar hierarchical nanowires (Fig. S8, ESI $\dagger$ ).

All the nanostructures synthesized by the covalently capped seed-mediated growth approach were highly branched, containing a plenty of tips, junctions, and sharp edges. These characteristics are favourable for SERS and catalytic applications. We therefore studied the SERS properties of HAuNSs and HAuNWs using one of the commonly used SERS analytes, Rhodamine 6G (R6G). The R6G deposited on a bare silicon substrate and $\mathrm{Au}$ nanosphere (AuNS)-modified substrates (the SEM image is shown in Fig. S4a, ESI $\dagger$ ) were tested for comparison. $1 \mu \mathrm{M}$ R6G could not be detected on the AuNSmodified substrate, whereas strong SERS signals were obtained from HAuNS- and HAuNW-modified substrates as shown in Fig. 2a. The Raman signal observed at $775 \mathrm{~cm}^{-1}$ is due to $\mathrm{C}-\mathrm{H}$ out-of-plane bending mode for R6G while 1182 $\mathrm{cm}^{-1}$ can be assigned to $\mathrm{C}-\mathrm{O}-\mathrm{C}$ stretching vibrations. The bands at $\sim 1360,1510,1575$, and $1650 \mathrm{~cm}^{-1}$ are due to the aromatic $\mathrm{C}-\mathrm{C}$ stretching vibrations. The Raman spectra agree well with the literature. ${ }^{\mathbf{4 2 , 4 3}}$ Strong Raman signals were obtained from the surface of hierarchical nanostructures with R6G as little as $1 \mathrm{nM}$ (Fig. 2b). The apparent enhancement factors $(\mathrm{AEF})^{\mathbf{2 6 , 4 4 - 4 6}}$ (see ESI $\dagger$ ) for the HAuNSs and HAuNWs were calculated to be $0.57 \times 10^{7}$ and $0.48 \times 10^{7}$, respectively. The average enhancement factors in this range are considered to be significantly high for SERS applications.

In summary, using covalently capped AuNPs as seeds, we have successfully demonstrated a new approach to delicately tune the morphology of hierarchical AuNPs. The hierarchically structured Au nanocrystals showed very high SERS enhancement. While the current study on Au-seeds covers a portion of the covalently capped seeded growth approach, the full implementation of the approach using other metal seeds should eventually lead to the rational synthesis of nanomaterials.

\section{Acknowledgements}

This work was financially supported by the Ministry of Education (MOE2012-T2-1-058) of Singapore.

\section{Notes and references}

1 A. M. Chen, O. Taratula, D. Wei, H. I. Yen, T. Thomas, T. J. Thomas, T. Minko and H. He, ACS Nano, 2010, 4, 3679-3688.

2 W. Luo, C. Zhu, S. Su, D. Li, Y. He, Q. Huang and C. Fan, ACS Nano, 2010, 4, 7451-7458.

3 L. Zhang, W. Niu and G. Xu, Nano Today, 2012, 7, 586-605.

4 J. N. Anker, W. P. Hall, O. Lyandres, N. C. Shah, J. Zhao and R. P. Van Duyne, Nat. Mater., 2008, 7, 442-453.

5 L. Guo, Y. Huang, Y. Kikutani, Y. Tanaka, T. Kitamori and D. H. Kim, Lab Chip, 2011, 11, 3299-3304.

6 B. Wu, L.-C. Chen, Y. Huang, Y. Zhang, Y. Kang and D.-H. Kim, Plasmonics, 2014, 1-7.

7 L. Guo, X. Zhou and D. H. Kim, Biosens. Bioelectron., 2011, 26, 2246-2251.

8 E. Boisselier and D. Astruc, Chem. Soc. Rev., 2009, 38, 17591782.

9 X. Huang, S. Neretina and M. A. El-Sayed, Adv. Mater., 2009, 21, 4880-4910.

10 P. Zijlstra, J. W. M. Chon and M. Gu, Nature, 2009, 459, 410413.

11 J. Ando, K. Fujita, N. I. Smith and S. Kawata, Nano Lett., 2011, 11, 5344-5348.

12 J. V. Jokerst, Z. Miao, C. Zavaleta, Z. Cheng and S. S. Gambhir, Small, 2011, 7, 625-633.

13 Y. Huang, A. R. Ferhan and D.-H. Kim, Nanoscale, 2013, 5, 7772-7775.

14 Y. Huang and D.-H. Kim, Nanoscale, 2012, 4, 6312-6317.

15 Y. Huang and D.-H. Kim, Nanoscale, 2011, 3, 3228-3232.

16 Y. Fang, S. Guo, C. Zhu, S. Dong and E. Wang, Langmuir, 2010, 26, 17816-17820.

17 A. Mohanty, N. Garg and R. Jin, Angew. Chem., Int. Ed., 2010, 49, 4962-4966.

18 B. Plowman, S. J. Ippolito, V. Bansal, Y. M. Sabri, A. P. O'Mullane and S. K. Bhargava, Chem. Commun., 2009, 5039-5041.

19 M. Bechelany, P. Brodard, J. Elias, A. Brioude, J. Michler and L. Philippe, Langmuir, 2010, 26, 14364-14371.

20 J. Fang, S. Du, S. Lebedkin, Z. Li, R. Kruk, M. Kappes and H. Hahn, Nano Lett., 2010, 10, 5006-5013.

21 W. Niu, L. Zhang and G. Xu, Nanoscale, 2013, 5, 3172-3181. 22 T. K. Sau and C. J. Murphy, J. Am. Chem. Soc., 2004, 126, 8648-8649.

23 C. H. Kuo and M. H. Huang, Langmuir, 2005, 21, 2012-2016. 24 O. M. Bakr, B. H. Wunsch and F. Stellacci, Chem. Mater., 2006, 18, 3297-3301.

25 L. Wang, C. H. Liu, Y. Nemoto, N. Fukata, K. C. W. Wu and Y. Yamauchi, RSC Adv., 2012, 2, 4608-4611.

26 M. Pradhan, J. Chowdhury, S. Sarkar, A. K. Sinha and T. Pal, J. Phys. Chem. C, 2012, 116, 24301-24313.

27 C. Gao, J. Goebl and Y. Yin, J. Mater. Chem. C, 2013, 1, 38983909.

28 Y. Yin and A. P. Alivisatos, Nature, 2005, 437, 664-670.

29 J. Watt, S. Cheong, M. F. Toney, B. Ingham, J. Cookson, P. T. Bishop and R. D. Tilley, ACS Nano, 2009, 4, 396-402. 
30 C. Gao, J. Vuong, Q. Zhang, Y. Liu and Y. Yin, Nanoscale, 2012, 4, 2875-2878.

31 X. Zhang, D. Li, L. Bourgeois, H. Wang and P. A. Webley, ChemPhysChem, 2009, 10, 436-441.

32 M. Ding, D. C. Sorescu, G. P. Kotchey and A. Star, J. Am. Chem. Soc., 2012, 134, 3472-3479.

33 K. Dawson, J. Strutwolf, K. P. Rodgers, G. Herzog, D. W. M. Arrigan, A. J. Quinn and A. O'Riordan, Anal. Chem., 2011, 83, 5535-5540.

34 A. Gole and C. J. Murphy, Chem. Mater., 2004, 16, 3633-3640.

35 A. A. Volkert, V. Subramaniam and A. J. Haes, Chem. Commun., 2011, 47, 478-480.

36 Y. Huang and D. H. Kim, Langmuir, 2011, 27, 13861-13867. 37 D. K. Smith and B. A. Korgel, Langmuir, 2008, 24, 644-649.

38 E. C. Cho, S. W. Choi, P. H. C. Camargo and Y. Xia, Langmuir, 2010, 26, 10005-10012.
39 W. Ni, X. Kou, Z. Yang and J. Wang, ACS Nano, 2008, 2, 677686.

40 K. Sohn, F. Kim, K. C. Pradel, J. Wu, Y. Peng, F. Zhou and J. Huang, ACS Nano, 2009, 3, 2191-2198.

41 Y. Huang, L. Wu, X. Chen, P. Bai and D.-H. Kim, Chem. Mater., 2013, 25, 2470-2475.

42 L. Jensen and G. C. Schatz, J. Phys. Chem. A, 2006, 110, 59735977.

43 S. Sil, N. Kuhar, S. Acharya and S. Umapathy, Sci. Rep., 2013, 3, 3336.

44 J. Parisi, L. Su and Y. Lei, Lab Chip, 2013, 13, 15011508.

45 A. Dandapat, S. Pramanik, S. Bysakh and G. De, J. Nanopart. Res., 2013, 15, 1-10.

46 W. Ren, S. Guo, S. Dong and E. Wang, J. Phys. Chem. C, 2011, 115, 10315-10320. 